\title{
Host Range of LPP Cyanophages
}

\author{
DAVID W. JOHNSON AND MALCOLM POTTS* \\ Cyanobacterial Research Group, Department of Biological Science, Florida State University, Tallahassee, Florida 32306
}

\begin{abstract}
We determined the sensitivities of 33 strains and variants of cyanobacteria to infection by the cyanophage LPP-1 archaetype, five LPP-1 serotypes, six LPP-2 serotypes, and 8 new LPP isolates. The LPP-1 archaetype and LPP-1 serotypes have different host ranges on strains of LPP group B.
\end{abstract}

Cyanophages LPP-1 and LPP-2 are classified as separate species within the genus Cyanopodovirus in the family Podoviridae (12). These phages were given the designation LPP as a consequence of their host range (members of the genera Lyngbya, Plectonema, and Phormidium) (13). In the taxonomic scheme of Rippka et al. (9), a provisional assignment to the LPP group has been made for filamentous cyanobacteria that fall within the broad confines of these three genera. A number of the strains in LPP group B are hosts for cyanophage LPP-1 and are considered to represent a single species $(9,16)$.

The LPP cyanophages are serologically unrelated but are considered to have the same host range $(12,15)$. However, hosts that are lysogenic for LPP-2 do not lyse when they are infected by LPP-2, and purified LPP-1 does not lyse S3 lysogens (6). In addition, the original serology determined for LPP-1 (ATCC 15581-P [ $^{\mathrm{T}}=$ type strain]) may have been partly dependent on contaminant bacteria (6). For example, archaetype LPP-1 was found to be incapable of establishing lysogeny, whereas the pure cyanophage, recovered by regrowth on an axenic host, had a host range and plaque morphology indicative of S3, a temperate cyanophage that is characterized by the formation of ringed plaques (5).

To evaluate these discrepancies in host range, we investigated the susceptibilities of 33 axenic strains and variants of cyanobacteria, including 20 that can be included in LPP group B on the basis of morphological criteria, to infection by the LPP-1 archaetype, five LPP-1 and six LPP-2 serotypes, and eight new LPP isolates.

(Some of the data were taken from an M.S. thesis submitted by D.W.J. to Florida State University.)

\section{MATERIALS AND METHODS}

Cyanobacteria. The generic names and in some cases the specific names of the cyanobacteria used in this study are as they appear in culture collection records.

(i) LPP group B. The following LPP group B (sensu Rippka et al. [9]) strains of filamentous, non-heterocystous cyanobacteria were obtained from R. Starr, University of Texas Culture Collection, Austin: Phormidium luridum UTEX 426; Phormidium faveolarum UTEX 427; Plectonema boryanum UTEX 482, UTEX 485, UTEX 487, UTEX 488, UTEX 581 (= PCC 6306 = ATCC 27894), UTEX 594 (= PCC $73110=$ ATCC 29407), UTEX 596, UTEX 597, UTEX 790, and UTEX 1542 (= PCC $6306=$ ATCC 27894); Plectonema calothricoides UTEX 598; Plectonema sp. strain UTEX 1541 (= PCC $6402=$ ATCC 27902); and Lyngbya keutzingii UTEX 1547 (= PCC 6506 = ATCC 29081).

An undesignated strain of Plectonema (designated strain FS180 in our laboratory) was obtained from M. M. Allen via

\footnotetext{
* Corresponding author.
}

E. I. Friedmann. The following three variants were derived from this strain: FS191 Ebr, FS192 Sds, and FS193 Str. These variants are resistant to $20 \mu \mathrm{g}$ of ethidium bromide per $\mathrm{ml}, 20 \mu \mathrm{g}$ of sodium dodecyl sulfate per $\mathrm{ml}$, and $20 \mu \mathrm{g}$ of streptomycin sulfate per $\mathrm{ml}$, respectively.

One strain, designated Phormidium sp. strain FS194 in our laboratory, was isolated from Lake Bradford in the vicinity of Tallahassee, Fla.

(ii) Non-LPP group. The following 13 strains not belonging to the LPP group of Rippka et al. (9) were included in the study for comparative purposes: Anabaena sp. strain ATCC 27899, Anabaena sp. strain FS195, Brachytrichia sp. strain FS196, Calothrix sp. strain FS197, Chroococcus sp. strains N41 and S24 (8), Dermocarpa sp. strain FS198, Eucapsis sp. strain FS199, Fischerella sp. strain FS200, Gloeocapsa sp. strain FS201, Hydrocoleum sp. strain FS202, Microcystis sp. strain FS203, and Nostoc commune UTEX 584. The strains with designations beginning with FS were obtained from the Carolina Biological Supply Co. and were subsequently purified in our laboratory.

Growth conditions. The isolation, purification, and maintenance of clonal axenic isolates were achieved by using established methods $(1,2,10)$. Specific conditions for growth have been described by Potts et al. $(7,8)$.

Cyanophages. LPP-1 archaetype ATCC 15581- $\mathrm{P}^{\mathrm{T}}$, five LPP-1 serotypes (serotypes AT, LC, WC, RSD, and SI) and six LPP-2 serotypes (serotypes GM, NI, WA, SCCF, SPI, and SA) were obtained from Robert S. Safferman (15). Stocks of these cyanophages were purified upon receipt by filtration (pore size, $0.5 \mu \mathrm{m}$; Millipore Corp.). Subsequent stocks were then prepared by using Plectonema boryanum strain UTEX 594 as the host (15).

Eight LPP isolates (designated A, B, C, D, E, M, N, and P) were purified from lysates of Plectonema sp. strain FS180 incubated with water samples obtained from two sewage treatment plants in Tallahassee, Fla. The methods used for isolation and purification have been described previously by Safferman and Morris $(11,13,14)$.

Host range and plaque morphology analyses. Double-layer and spot assay methods were used to assess the host ranges of cyanophage isolates $(3,6)$. For the latter method, the concentration of cyanophages used was approximately $10^{8}$ phage per drop. Cyanophage titers were determined by plaque counting, using Plectonema sp. strain FS180 as the sensitive host.

Tests for lysogeny. Three assays were used to test for lysogeny. In the double-layer assay, the strain or variant to be tested was incorporated in an agar solution and poured as a thin layer over a solid base layer of a sensitive host, Plectonema sp. strain FS180. For the spot test, a $50-\mu 1$ sample of a liquid suspension of the strain to be tested was placed onto the surface of a layer of Plectonema sp. strain FS180. In the third assay, the surface of an agar plate was 
inoculated with Plectonema sp. strain FS180 in a single narrow streak. A second streak was made to form a cross, using an inoculum of the strain to be tested. In each assay, zones of lysis or plaques were considered positive indications of lysogeny.

Cyanophage adsorption assays. A cyanophage stock of known titer was added to a culture, and the mixture was shaken at $25^{\circ} \mathrm{C}$ under subdued light. Portions of the suspension were removed at intervals, the filaments (with adsorbed cyanophage particles) were removed by low-speed centrifugation, and the supernatant was analyzed in a plaque assay to determine the titer of unadsorbed particles.

\section{RESULTS}

None of the 13 strains belonging to the non-LPP group was infected by any of the cyanophage isolates. Among the other 20 strains and variants of non-heterocystous filamentous cyanobacteria tested, Plectonema sp. strain FS180, variants FS191 Ebr, FS192 Sds, and FS193 Str, and strains UTEX 426, UTEX 427, UTEX 482, UTEX 485, UTEX 487, UTEX 488, UTEX 581, UTEX 594, UTEX 596, UTEX 597, and UTEX 790 expressed a persistent phenotype of sensitivity to all viral isolates during numerous trials over a period of 2 years. For these strains and variants, lysis occurred within 2 to 7 days after infection. Cyanophage titers (as measured by the plaque assay) ranged from approximately $10^{7}$ to $10^{10}$ plaque-forming units per $\mathrm{ml}$, and this range in variation was found in lysates of cultures incubated at 25 as well as $34^{\circ} \mathrm{C}$. Infection of sensitive hosts was prevented at incubation temperatures greater than $40^{\circ} \mathrm{C}$. No plaques developed within the $2 \mathrm{~h}$ after infection of overlayer plates of sensitive strains, and plaques rarely appeared within $24 \mathrm{~h}$. Phenomena associated with bacteriocin (cyanocin) activities were not observed. Ringed plaques were never seen. On several occasions, plaques with single centers of infection were noted. Isolation of viral particles from these plaques and subsequent infection of the same host did not give rise to the same plaque morphology (i.e., with single centers of infection).

L. keutzingii strain UTEX 1547 was not infected by any of the cyanophage isolates tested by using a wide range of multiplicity of infection.

Plectonema calothricoides UTEX 598 was sensitive to the LPP-1 archaetype but remained persistently insensitive to LPP-1 and LPP-2 serotypes, as well as the isolates of our laboratory, over a period of 2 years. No visible change in a culture of Plectonema calothricoides UTEX 598 occurred during infection with these cyanophages. Nevertheless, studies with cyanophage isolate $\mathrm{A}$ demonstrated that particles adsorb to this host and sensitive Plectonema sp. strain FS180 with equal efficiency.

Plectonema sp. strain UTEX 1541 and Plectonema boryanum UTEX 1542 showed the same "insensitive" response with cyanophage isolates other than the LPP-1 archaetype. Overlayers showed only a slight discoloration, without lysis, after 5 to 7 days of infection. For these strains and also for strain UTEX 598, all tests for lysogeny were negative.

\section{DISCUSSION}

LPP group B was proposed as a provisional assemblage of non-heterocystous, unbranched filamentous cyanobacteria that are characterized by isodiametric or cylindrical cells, transcellular or intercellular breakage, and lack of polar gas vesicles (9). The taxonomic status of this group and the number of genera contained within it are, at this time, undetermined. A total of 11 strains that were sensitive to infection by all 20 cyanophage isolates tested in this study are known to share a high degree of relatedness, as evidenced by deoxyribonucleic acid-deoxyribonucleic acid hybridization studies (range of relatedness with strain UTEX 594 as the reference, 95 to $112 \%$; guanine-plus-cytosine content range, 45 to $46 \mathrm{~mol} \%)(4,9,16,17)$. Our data support the view that these strains represent a single taxonomic group. At this time, the generic assignment and its limits remain undetermined. Although the utility of plasmids in cyanobacterial taxonomy has not yet been assessed critically, it is significant that these 11 strains all have identical plasmid profiles $(7,7 a)$.

Because of its insensitivity to all of the cyanophages tested, the strain designated L. keutzingii UTEX 1547 is clearly distinct. Rippka et al. (9) included this strain (designated strain PCC 6506) in the genus Oscillatoria on the basis of the presence of disk-shaped cells without deep constriction and motile trichomes.

Of the three morphologically similar strains that are sensitive to the LPP-1 archaetype but are insensitive to other LPP-1 and LPP-2 serotypes, strain UTEX 1542 (= PCC 6306) is listed as equivalent to strain UTEX 581 (included in the group of sensitive strains above), strain UTEX 598 (guanine-plus-cytosine content, $45 \mathrm{~mol} \%$ ) shares $99 \%$ homology with strain UTEX 594, and strain UTEX 1541 (= PCC 6402) (guanine-plus-cytosine content, $46 \mathrm{~mol} \%$ ) shares $103 \%$ homology with strain UTEX $594(9,16$; see above). On the basis of these criteria, one would expect to include these three strains within the single taxonomic group indicated above. Nevertheless, Plectonema sp. strain UTEX 1541 has a genome molecular weight $\left(3.99 \times 10^{9}\right)$ that is significantly different from the genome molecular weights of strains UTEX 581, UTEX 594, and UTEX 1542 (range, $4.78 \times 10^{9}$ to $\left.5.15 \times 10^{9}[2]\right)$. The insensitivity of the three strains to all of the cyanophage isolates except LPP-1 is significant. All attempts to demonstrate lysogeny in these strains have been negative, although our assays would not detect defective lysogeny. Nevertheless, LPP-1 is known to be incapable of lysogeny $(5,6)$, and in this respect it is difficult to account for the insensitivity of these three strains to infection by LPP-1 serotypes, particularly isolate AT, which has identical neutralization kinetics with LPP-1 archaetype antiserum. In this regard, archaetypal cyanophage LPP-1 is distinct from other LPP-1 serotypes, a point first suggested by Kraus (6).

The LPP cyanophages were designated according to their apparent host range (species belonging to the genera Lyngbya, Plectonema, and Phormidium) (13). The results of this study confirm previous observations that the host range of these cyanophages is restricted to certain filamentous, unbranched, non-heterocystous strains (14). However, the LPP designation reflects inadequacies in cyanobacterial taxonomy rather than a true polygeneric host range (12). In fact, Stam (16) has recommended that the genera Lyngbya, Plectonema, and Phormidium be merged into a single genus. The validity of this recommendation can be assessed only after a thorough characterization of additional strains and isolates that would be given one of these three generic assignments based on current taxonomic criteria.

Our data indicate the presence of distinct groupings within the taxonomic group that encompasses LPP strains with a high degree of deoxyribonucleic acid relatedness and mutual sensitivity to the LPP-1 archaetype. The fact that the LPP-1 archaetype and LPP-1 serotypes have different host ranges and the less restrictive nature of the LPP-1 archaetype should be considered very carefully in situations where 
susceptibility to LPP cyanophages is used routinely as a discriminating feature within a taxon.

\section{ACKNOWLEDGMENTS}

We thank R. S. Safferman for gifts of the LPP-1 archaetype and other cyanophages and M. M. Allen for helpful discussions relating to Plectonema sp. strain FS180. We are grateful to E. I. Friedmann for critically reading the manuscript.

This study was supported in part by grant PCM 8203709 from the National Science Foundation.

\section{LITERATURE CITED}

1. Allen, M. M. 1968. Simple conditions for the growth of unicellular blue-green algae on plates. J. Phycol. 4:1-4.

2. Herdman, M., M. Janvier, R. Rippka, and R. Y. Stanier. 1979. Genome size of cyanobacteria. J. Gen. Microbiol. 111:73-85.

3. Hu, N.-T., T. Thiel, T. H. Giddings, Jr., and C. P. Wolk. 1981. New Anabaena and Nostoc cyanophages from sewage settling ponds. Virology 114:236-246.

4. Kelly, M. L., and D. B. Cowie. 1971. DNA-DNA hybridization studies of blue-green algae. Carnegie Inst. Washington Yearb. 71:276-281.

5. Kraus, M. P. 1974. Host range and plaque morphology of blue-green algal viruses. Seagrant Publication SG-DEL-1-74. University of Delaware, Newark.

6. Kraus, M. P. 1980 . Host-range, plaque morphology studies of cyanophage LPP-1. J. Phycol. 17:186-191.

7. Potts, M. 1984. Isolation of plasmids from cyanobacteria. Focus 6:4-6.

7a.Potts, M. 1984. Distribution of plasmids in cyanobacteria of the LPP group. FEMS Microbiol. Lett. 24:351-354.

8. Potts, M., R. Ocampo-Friedman, M. A. Bowman, and B. Tözún.
1983. Chroococcus S24 and Chroococcus N41 (cyanobacteria): morphological, biochemical and genetic characterization and effects of water stress on ultrastructure. Arch. Microbiol. 135:81-90.

9. Rippka, R., R. Deruelles, J. B. Waterbury, M. Herdman, and R. Y. Stanier. 1979. Generic assignments, strain histories and properties of pure cultures of cyanobacteria. J. Gen. Microbiol. 111:1-61.

10. Rippka, R., J. B. Waterbury, and R. Y. Stanier. 1981. Isolation and purification of cyanobacteria: some general principles, $\mathrm{p}$. 212-220. In M. P. Starr, H. Stolp, H. G. Trüper, A. Balows, and H. G. Schlegel (ed.), the prokaryotes, vol. 1. SpringerVerlag, Berlin.

11. Safferman, R. S. 1973. Phycoviruses. Bot. Monogr. 9:214-237.

12. Safferman, R. S., R. E. Cannon, P. R. Desjardins, B. V. Gromov, R. Haselkorn, L. A. Sherman, and M. Shilo. 1983. Classification and nomenclature of viruses of cyanobacteria. Intervirology 19:61-66.

13. Safferman, R. S., and M. E. Morris. 1963. Algal virus: isolation. Science 140:679-680.

14. Safferman, R. S., and M. E. Morris. 1967. Observations on the occurrence, distribution, and seasonal incidence of blue-green algal viruses. Appl. Microbiol. 15:1219-1222.

15. Safferman, R. S., M. E. Morris, L. A. Sherman, and R. Haselkorn. 1969. Serological and electron microscopic characterization of a new group of blue-green algal viruses (LPP-2). Virology 39:775-780.

16. Stam, W. T. 1980. Relationship between a number of filamentous blue-green algal strains (Cyanophyceae) revealed by DNADNA hybridization. Algol. Stud. 25:351-374.

17. Stam, W. T., and G. Venema. 1977. The use of DNA-DNA hybridization for determination of the relationship between some blue-green algae (Cyanophyceae). Acta Bot. Neerl. 26:327-342. 